\title{
A particle emission region size in multiparticle production process
}

\author{
Ts. Baatar ${ }^{1}$ \\ Institute of Physics and Technology, MAS, Ulaanbaatar, Mongolia \\ Ulaanbaatar University, Ulaanbaatar, Mongolia \\ E-mail: baatar1945@yahoo.com
}

\section{G.Sharkhuu, B. Otgongerel}

Institute of Physics and Technology, MAS, Ulaanbaatar, Mongolia

E-mail: otgongerelb@gmail.com

\section{A.I. Malakhov}

Joint Institute for Nuclear Research, Dubna, Russia

E-mail: malakhovelhe.jinr.ru

In this paper we have obtained the formula which determines the particle emission region size and corresponding distributions are shown for the secondary negative pions and protons from $\pi^{-} C$ interactions at $40 \mathrm{GeV} / \mathrm{c}$.

XXII International Baldin Seminar on High Energy Physics Problems

15-20 September 2014

JINR, Dubna, Russia

\section{${ }^{1}$ Speaker}




\section{Introduction}

An investigation of multiparticle production processes of the secondary particles produced in hadron-hadron (hh), hadron-nucleus (hA) and nucleus-nucleus (AA) interactions at high energies and large momentum transfers is very important for understanding the strong interaction mechanism and inner quark-gluon structure of nuclear matter.

A determination of the particle production size of the secondary particles of above mentioned interactions is one of the key problems in the studies of high energy physics. A correct estimation of this parameter (r) gives us the possibility to calculate the volume, mass and energy densities and to establish the main characteristics of the phase transition process, to distinguish the different mechanisms of the secondary particle's production and so on.

During the last years the collective phenomena such as the cumulative particle production, the production of nuclear matter with high densities, the phase transition from the hadronic matter to the quark-gluon plasma state, the state of the color superconductivity is widely discussed in the literature [1-7].

According to the different ideas and models, if exist these phenomena in the nature then they should be reflected to the dynamics of interaction process and will be observed in hA and AA interactions at high energies and large momentum transfers. We would like to stress that in hA and AA collisions in difference from hh collisions the secondary particles may be produced in the regions kinematically not allowed to hh interactions.

\section{A determination of the particle emission region size}

The cumulative number $n_{c}$ in the fixed target experiment is determined by the next formula [1],

$$
n_{c}=\frac{\left(P_{a} \cdot P_{c}\right)}{\left(P_{a} \cdot P_{b}\right)}=\frac{E_{c}-\beta_{a} \cdot P_{c}^{I I}}{m_{p}} \approx \frac{E_{c}-P_{c}^{I I}}{m_{p}}
$$

Where $P_{a}, P_{b}$ and $P_{c}$ are the four dimensional momenta of incident, target and considering secondary particles. $E_{c}$ and $P_{c}^{I I}$ are the energy and longitudinal momentum of the secondary particle, $\beta_{a}$ is the velocity of the incident particle and $m_{p}$ is the proton mass.

The variable $n_{c}$ is connected with the four momentum transfer $t$ by the next formula,

$$
t=-Q^{2}=-\left(P_{a}-P_{c}\right)^{2} \cong 2 E_{a} \cdot m_{p} \cdot n_{c}-\left(m_{a}^{2}+m_{c}^{2}\right)
$$

Where $E_{a}$ and $m_{a}$ are the energy and mass of the incident particle, $m_{c}$ is the mass of the considering secondary particle.

A determination of the particle emission region size $r$ of secondary particles is very important in the study of multiparticle production dynamics.

It is well known that the particle emission region size $r$ is inversely proportional to the momentum transfer $Q$

$$
r \sim \frac{1}{Q}
$$


Assuming that dependence (3) is correct and then including a coefficent of proprotionality $k_{0}$ we obtain the next equation,

$$
r=\frac{k_{0}}{Q}=\frac{k_{0}}{\sqrt{2 E_{a} \cdot m_{p} \cdot n_{c}-\left(m_{a}^{2}+m_{c}^{2}\right)}}
$$

Now we choose the condition $\left(n_{c}=1\right)$, this procedure gives us the possibility to simplify the formula (4) and to determine the value of the parameter $r$ corresponding to this condition $\left(n_{c}=1\right)$. We note that the condition $n_{c}=1$ means that the value of the target mass which is required for producing of the given secondary particle is equal to proton mass $m_{p}$. In this case the formula (4) is written in the next form,

$$
k_{0}=r \cdot \sqrt{2 E_{a} \cdot m_{p}-\left(m_{a}^{2}+m_{c}^{2}\right)}
$$

We note that as mentioned above at $n_{c}=\frac{E_{c}-\beta_{a} \cdot P_{c}^{I I}}{m_{p}}=\frac{m_{p}}{m_{p}}=1$, the parameter $r$ is equal to the Compton wavelength of proton $\chi_{c}^{P}$, in other words,

$$
r=\lambda_{c}^{P}=\frac{1}{m_{p}}=0.21 \mathrm{fm}
$$

Inserting the formula (6) to equation (5) the coefficient of proportionality $\mathrm{k}_{0}$ is determined by the next formula,

$$
k_{0}=\lambda_{c}^{P} \cdot \sqrt{2 E_{a} \cdot m_{p}-\left(m_{a}^{2}+m_{c}^{2}\right)}=\frac{\sqrt{2 E_{a} \cdot m_{p}-\left(m_{a}^{2}+m_{c}^{2}\right)}}{m_{p}}
$$

Fig. 1 presents the dependence of the parameter $k_{0}$ on $\sqrt{S_{h N}}=\sqrt{2 E_{a} \cdot m_{p}}$.

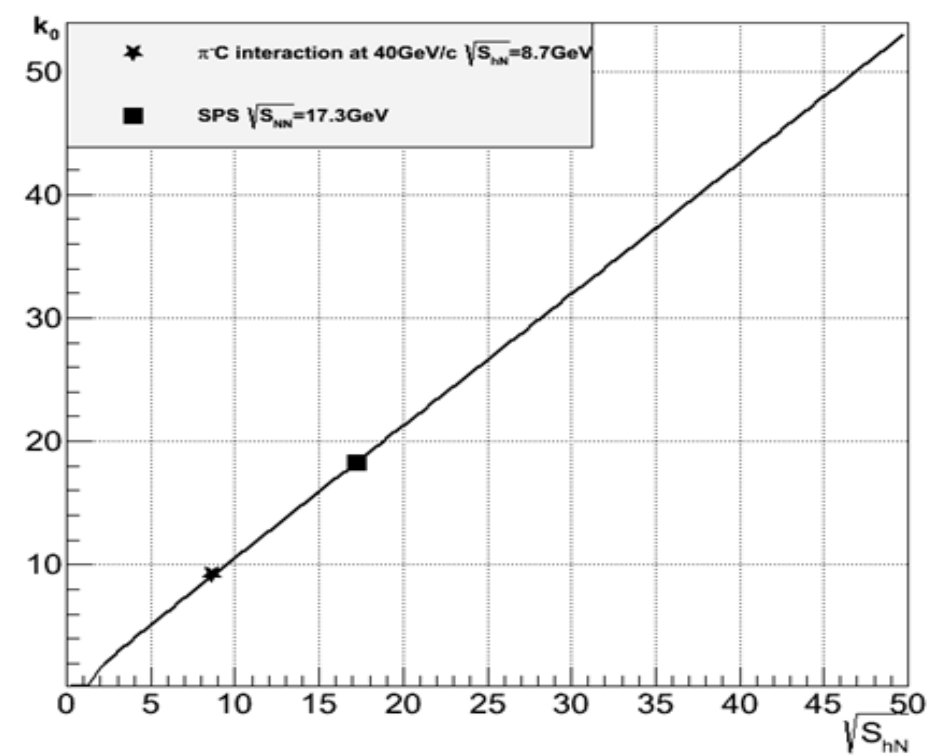

Figure 1 shows the dependence of the parameter $k_{0}$ on $\sqrt{S_{h N}}$

From this figure we see that the dependence of the parameter $k_{0}$ on $\sqrt{S_{h N}}$ at high energies $\left(2 E_{a} m_{p} \gg\left(m_{a}^{2}+m_{c}^{2}\right)\right)$ is described by the linear law and at low energies mass term $\left(m_{a}^{2}+m_{c}^{2}\right)$ gives a small deviation from the linear law. 
Receiving the formula (7) for the parameter $\mathrm{k}_{0}$, of course, we have the possibility to obtain the formula which determines the particle emission region size $r$.

Inserting the formula (7) to the formula (4) we are obtained the next formula for the parameter $\mathrm{r}$,

$$
r=\frac{1}{m_{p} \sqrt{1+\frac{2 E_{a} m_{p}\left(n_{c}-1\right)}{2 E_{a} m_{p}-\left(m_{a}^{2}+m_{c}^{2}\right)}}}
$$

From formula (8) we see that the dependence of the parameter $r$ on $\sqrt{S_{h N}}=\sqrt{2 E_{a} m_{p}}$ at high energies $\left(S_{h N} \gg\left(m_{a}^{2}+m_{c}^{2}\right)\right)$ is slow and so the formula (8) is mainly determined by the next formula,

$$
r=\frac{1}{\sqrt{n_{c}} \cdot m_{p}}=\frac{\lambda_{c}^{P}}{\sqrt{n_{c}}}=\frac{0.21 \mathrm{fm}}{\sqrt{n_{c}}}
$$

From the formula (9) we see that the parameter $\mathrm{r}$ is fully determined by the Compton wavelength of proton $\lambda_{c}^{P}$, the cumulative number $\mathrm{n}_{\mathrm{c}}$ and $\sqrt{S_{h N}}$. So the determination of the numerical value of the variable $n_{c}$ gives us the possibility to obtain the numerical value of the particle emission region size $r$ for the every secondary particle produced in collisions of interacting particles and nuclei at high energies

From formula (4) the parameter $k_{0}$ is written in the next form,

$$
k_{0}=r_{i} \sqrt{S_{h N} \cdot n_{c}^{i}}
$$

The dependence of the parameter $k_{0}$ on the variable $n_{c}$ was shown for Fig.2 that the parameter $k_{0}$ do not depend on the variable $n_{c}$ at fixed energy $\sqrt{S_{h N}}$.

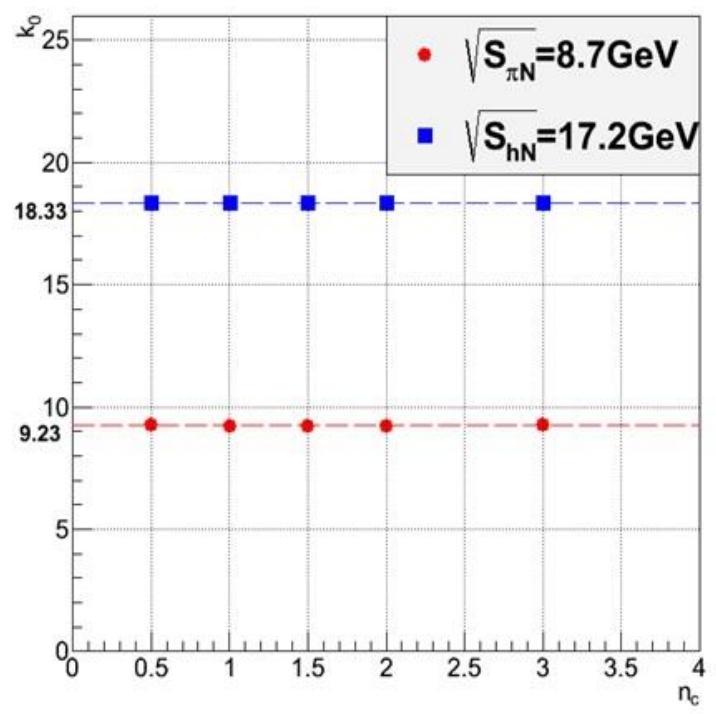

Fig.2 Dependence of the parameter $k_{0}$ on the variable $n_{c}$. 
The dependence of the parameter $r^{2}$ on the variable $n_{c}$ calculated by the formula (8) is presented on Fig.3. This dependence do not depend on the type of the secondary particles and $\sqrt{S_{h N}}$.

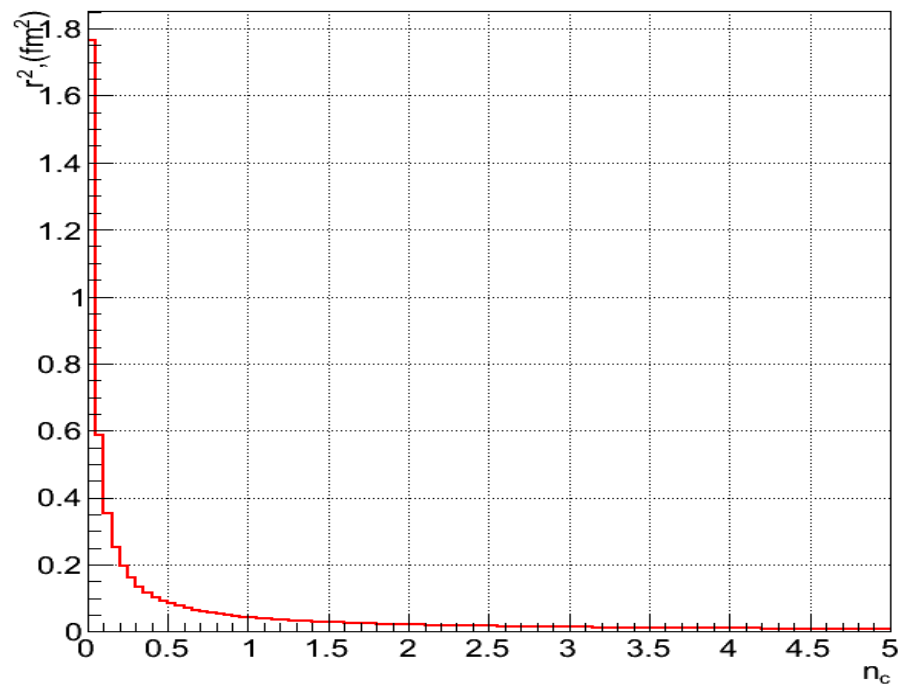

Fig.3 Dependence of the parameter $r$ on $n_{c}$

\section{In the case of $\pi^{-} C$ interactions at $40 \mathrm{GeV} / \mathrm{C}$}

\section{1 $\pi^{-}+C \rightarrow \pi^{-}+X$ analysis}

We would like to stress that the leading $\pi^{-}$mesons may give some effect to the experimental result. So we are considered for $\pi^{-}$mesons two cases with and without leading particles. The angular distribution of $\pi^{-}$mesons with momentum was shown on Fig.4. Fromthis distribution $\pi^{-}$mesons with momentum and scattering angle $\theta<4^{\circ}$ are regarded as leading particles. 1182 $\pi^{-}$mesons are excluded from total 30162 pions as leading particles.

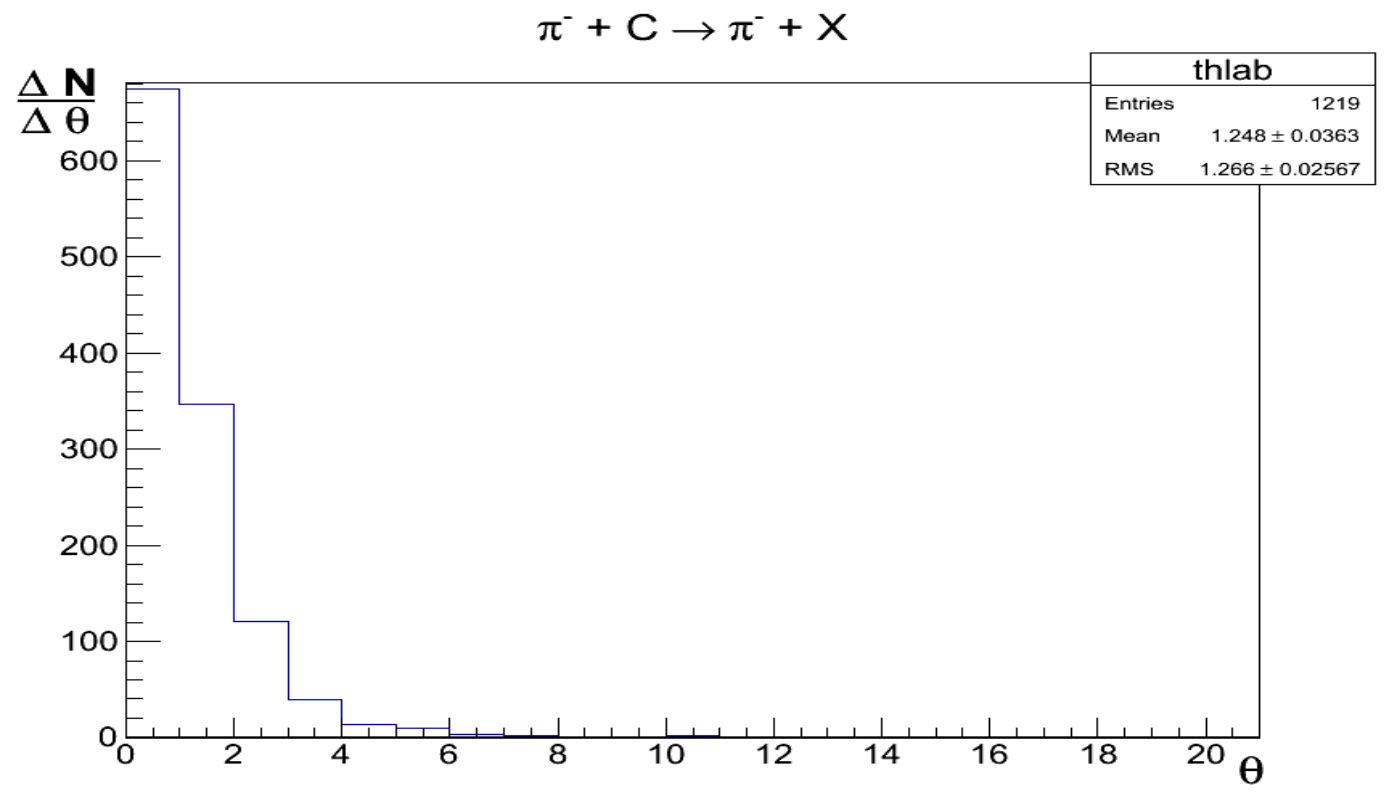

Fig.4. Angular distribution of $\pi^{-}$mesons with momentum 


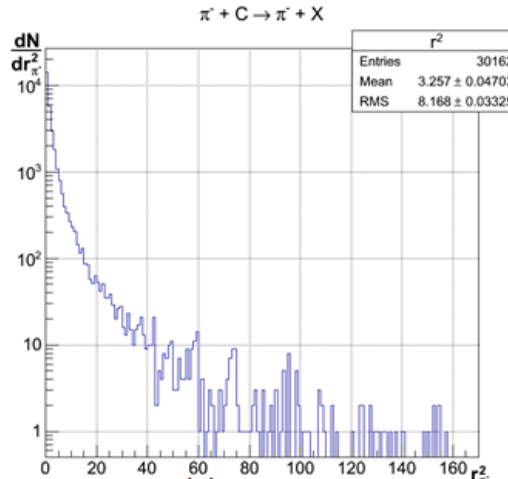

(a)

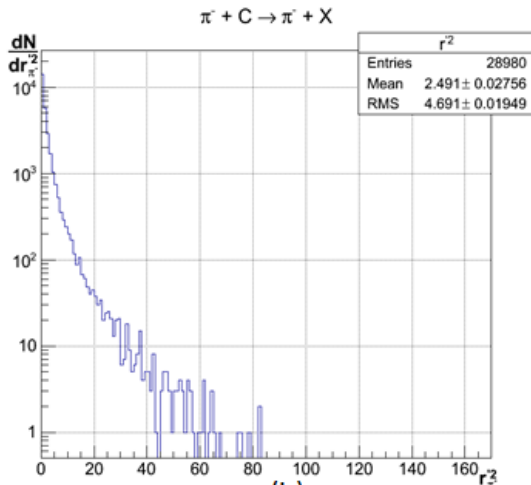

(b)

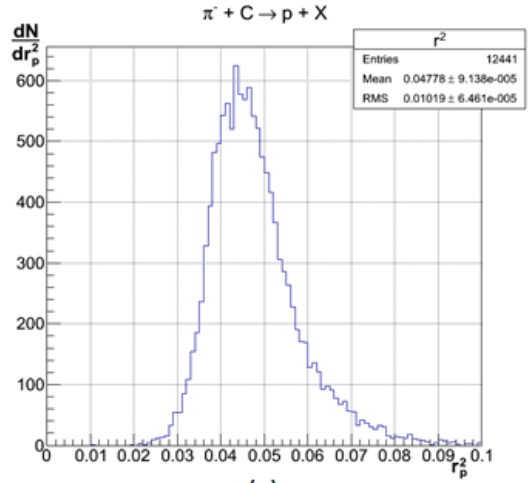

(c)

Fig.5 a, b, c. Distribution on $r^{2}$ for $\pi^{-}$mesons (a, b) and protons (c) from $\pi^{-} C$ interactions at $40 \mathrm{GeV} / \mathrm{c}$

The experimental distributions on the parameter $r^{2}$ calculated by the formula (8) for all $\pi^{-}$ mesons $r_{\pi^{-}}^{2}, \pi^{-}$mesons without leading particles $\left(r_{\pi^{-}}^{\prime}\right)^{2}$ and protons $r_{p}^{2}$ from $\pi^{-} C$ interactions at $40 \mathrm{GeV} / \mathrm{c}$ are shown on Fig.5 $(\mathrm{a}, \mathrm{b}, \mathrm{c})$. The next average values are obtained:

$$
\begin{gathered}
<r>_{\pi^{-}}=\sqrt{\left\langle r^{2}>_{\pi^{-}}\right.}=(1.805 \pm 0.026) \mathrm{fm} \\
<r^{\prime}>_{\pi^{-}}=\sqrt{\left\langle\left(r^{\prime}\right)^{2}>_{\pi^{-}}\right.}=(1.578 \pm 0.017) \mathrm{fm} \\
<r>_{p}=\sqrt{\left\langle r^{2}>_{p}\right.}=(0.218 \pm 0.002) \mathrm{fm} \\
\frac{\left\langle r>_{\pi^{-}}\right.}{\left\langle r>_{p}\right.}=\frac{1.805}{0.218}=8.2, \frac{\left\langle r>_{\pi^{-}}\right.}{\left\langle r>_{p}\right.}=\frac{1.578}{0.218}=7.2
\end{gathered}
$$

From this analysis we see that the average value of the parameter $\langle r\rangle_{\pi^{-}}$for $\pi^{-}$mesons is $7 \div 8$ times greater than the case for protons $\left\langle r>_{p}\right.$. We note that ratios of $\langle r\rangle_{\pi^{-}},\left\langle r^{\prime}\right\rangle_{\pi^{-}}$ to $\langle r\rangle_{p}$ obtained within our approach is in reasonable consistence with a theoretical prediction referred in [8] as follows:

$$
\lambda_{c}^{\pi} \cong 7 \cdot \lambda_{c}^{p}
$$

Where $\lambda_{c}^{\pi}$ and $\lambda_{c}^{p}$ are the Compton wavelengths of pion and proton.

Average values of the parameters $\left\langle r^{2}\right\rangle_{\pi^{-}},\langle r\rangle_{\pi^{-}}$for the all secondary $\pi^{-}$mesons and $\pi^{-}$mesons in three different regions on the variable $n_{c}$ are presented in Table 1.

Corresponding values without leading $\pi^{-}$mesons are presented in Table 2.

Table 1

\begin{tabular}{|c|c|c|c|c|}
\hline \multicolumn{5}{|c|}{$\pi^{-}+C \rightarrow \pi^{-}+X$} \\
\hline & All $\pi$-mesons & $\begin{array}{c}\pi^{-} \text {-mesons } \\
\text { with } \mathrm{n}_{0} \leq 0.07\end{array}$ & $\begin{array}{c}\pi^{-} \text {-mesons } \\
0.07<\mathrm{n}_{0}<0.5\end{array}$ & $\begin{array}{c}\pi^{-} \text {-mesons } \\
\mathrm{n}_{0}>0.5\end{array}$ \\
\hline$N_{\pi^{-}}$ & 30162 & 19702 & 9879 & 581 \\
\hline$\left\langle r^{2}\right\rangle_{\pi^{-}}\left(\mathrm{fm} \mathrm{m}^{2}\right)$ & $3.122 \pm 0.041$ & $4.614 \pm 0.059$ & $0.331 \pm 0.002$ & $0.06146 \pm 0.0009$ \\
\hline$\langle r\rangle_{\pi^{-}}(\mathrm{fm})$ & $1.769 \pm 0.023$ & $2.148 \pm 0.028$ & $0.575 \pm 0.003$ & $0.2479 \pm 0.0036$ \\
\hline
\end{tabular}


Table 2

\begin{tabular}{|c|c|c|c|c|}
\hline \multicolumn{6}{|c|}{$\pi^{-}+C \rightarrow \pi^{-}+X$} \\
\hline & $\begin{array}{c}\pi^{-} \text {-mesons } \\
\text { without leading } \\
\text { particles }\end{array}$ & $\begin{array}{c}\pi^{-} \text {-mesons } \\
\text { with } \mathrm{n}_{\mathrm{c}} \leq 0.07\end{array}$ & $\begin{array}{c}\pi^{-} \text {-mesons } \\
0.07<\mathrm{n}_{\mathrm{c}}<0.5\end{array}$ & $\begin{array}{c}\pi^{-} \text {-mesons } \\
\mathrm{n}_{\mathrm{c}}>0.5\end{array}$ \\
\hline$N_{\pi^{-}}$ & 28980 & 18522 & 9877 & 581 \\
\hline$\left\langle r^{\prime 2}\right\rangle_{\pi^{-}}\left(\mathrm{fm}^{2}\right)$ & $2.491 \pm 0.0276$ & $3.72 \pm 0.040$ & $0.331 \pm 0.002$ & $0.06146 \pm 0.0009$ \\
\hline$\left\langle r^{\prime}\right\rangle_{\pi^{-}}(\mathrm{fm})$ & $1.5783 \pm 0.017$ & $1.929 \pm 0.021$ & $0.575 \pm 0.003$ & $0.2479 \pm 0.0036$ \\
\hline
\end{tabular}

\section{$3.2 \pi^{-}$mesons in three different $n_{c}$ regions}

The dependence of the effective temperature $T$ on the variable $n_{c}$ is presented on Fig.6. This dependence was taken from publication [1]. From this figure we see that with increasing $n_{c}$ the effective temperature $T$ is increasing in the beginning until $n_{c} \leq 0.07$ and then in interval $(0.07<$ $n_{c}<0.5$ ) the parameter $T$ remains practically constant on the level $T=0.220 \div 0.230 \mathrm{GeV}$ and then from the point $n_{c}>0.5$ is increasing again.

The corresponding values of the parameter $r$ at breaking points of the dependence are shown by arrows on this figure.

The proton charge radius usually is determined from the elastic electron-proton scattering [9] as follows:

$$
R_{p}=0.8775 \pm 0.0051 \mathrm{fm}
$$

The value of the parameter $r$ calculated by formula ( 8$)$ at the first critical point $\left(n_{c}=0.071\right)$ which gives the beginning of the plateau on parameter $T$ is obtained equal to $0.79 \mathrm{fm}$ (see Fig.6.). We note that this value is compatible with the charge radius of proton mentioned above. So, if the secondary $\pi^{-}$mesons are produced at the smaller or approximately equal distances to the charge radius of proton then these particles begin to actively participate in the equilibrium process (or phase transition).

The plateau on parameter $T$ is continued to $\langle r\rangle_{\pi^{-}} \leq 0.297 \mathrm{fm}$, so the negative pions produced at the distances $\langle r\rangle_{\pi^{-}} \geq 0.297 \mathrm{fm}$ give the main contribution to the pure quarkgluon plasma state.

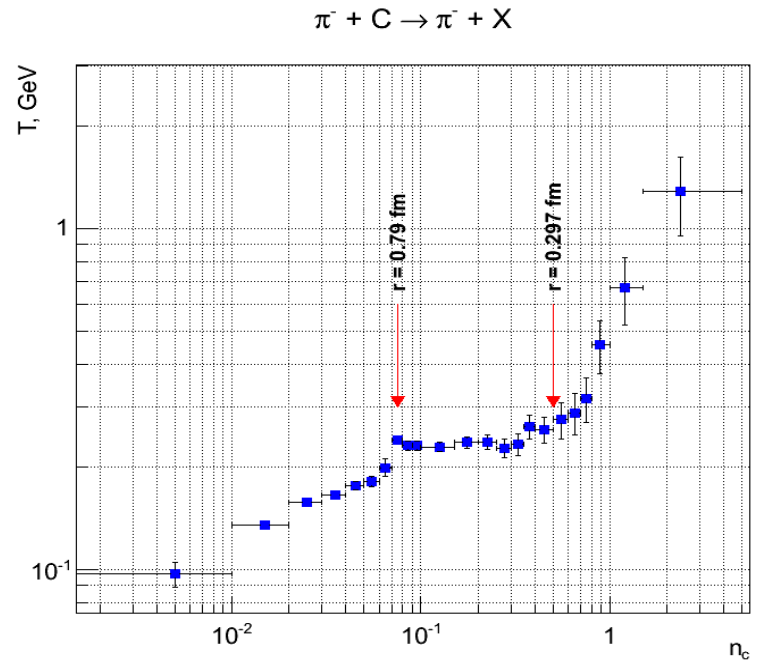

Fig. 6. The effective temperature $T$ of the secondary $\pi^{-}$mesons as a function of the variable $n_{c}$. This figure was taken from paper [1]. 
Figure $7(\mathrm{a}, \mathrm{b} \mathrm{c})$ shows distributions on $r^{2}$ for $\pi^{-}$mesons in the above mentioned three different phase transition regions. We would like to stress that the particle production region size $r$ is essentially different in every region.
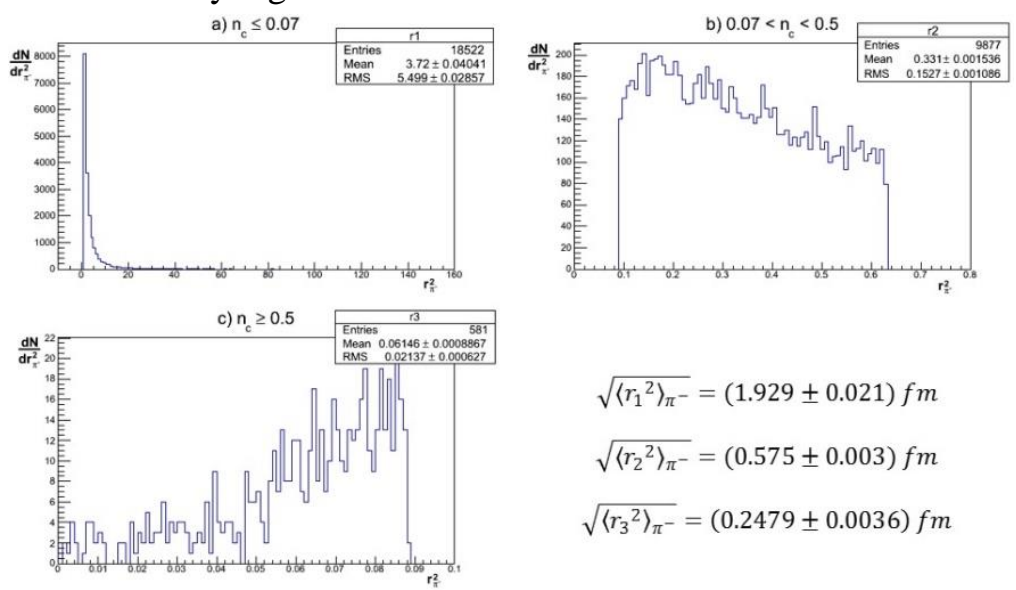

$$
\begin{aligned}
\sqrt{\left\langle r_{1}^{2}\right\rangle_{\pi^{-}}} & =(1.929 \pm 0.021) \mathrm{fm} \\
\sqrt{\left\langle r_{2}^{2}\right\rangle_{\pi^{-}}} & =(0.575 \pm 0.003) \mathrm{fm} \\
\sqrt{\left\langle r_{3}^{2}\right\rangle_{\pi^{-}}} & =(0.2479 \pm 0.0036) \mathrm{fm}
\end{aligned}
$$

Fig.7. a, b, c. Distribution on $r^{2}$ for $\pi^{-}$mesons in different phase transition regions

\section{$3.3 \pi^{-}+\boldsymbol{C} \rightarrow \boldsymbol{P}+\boldsymbol{X}$ analysis}

Figure 8 shows the dependence of the effective temperature $T$ on the variable $n_{c}$ for the secondary protons from $\pi^{-} C$ interactions. From this figure we see that with increasing $n_{c}$, this dependence is divided into two parts, where temperature $T$ is remained practically constant on the level $T \approx 50 \mathrm{MeV}$ in the $n_{c} \cong 0.5 \div 1.2$ interval and $T$ is increased in the region $n_{c}>1.2$.

The corresponding values of the parameter $r$ on the breaking points of the dependence are shown by arrows on this figure.

We note that the numerical values of the parameter $T$ which gives the plateau for $\pi^{-}$mesons $(\sim 230 \mathrm{MeV})$ and for $(\sim 50 \mathrm{MeV})$ are essentially different but, the behaviors of the dependence of parameter $T$ on the variable $n_{c}$ are the same in both cases.

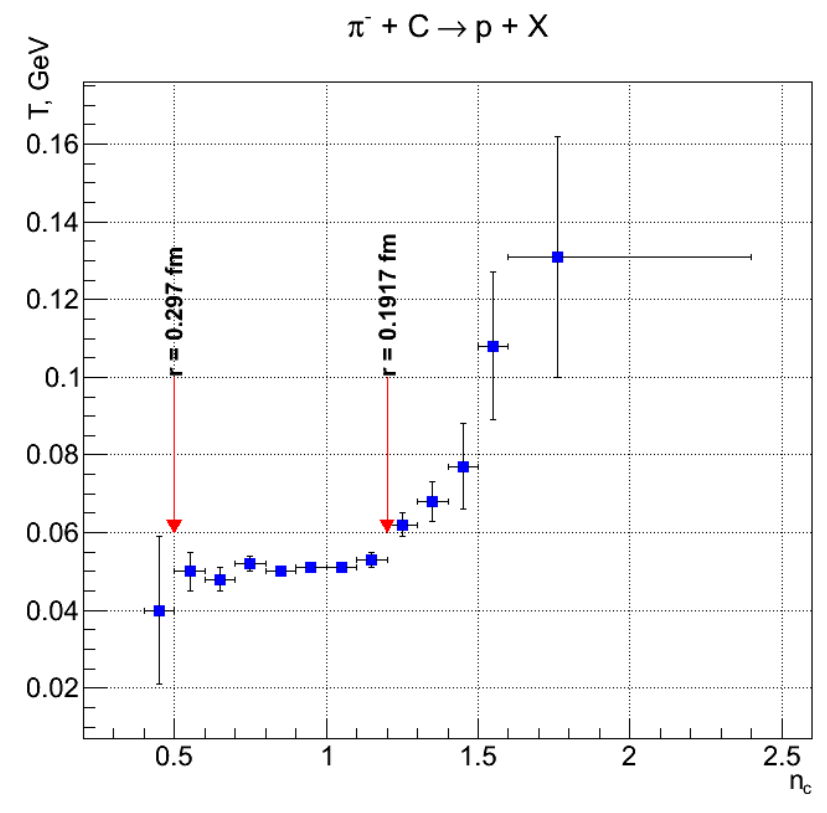

Fig.8. The effective temperature $T$ of the secondary protons as a function of the variable $n_{c}$. This figure was taken from paper [1]. 
Fig. 9 a, b shows distributions on parameter $\mathrm{r}^{2}$ in these two regions. We would like to note that the protons from $\pi^{-} C$ interactions at $40 \mathrm{GeV} / \mathrm{c}$ are produced at comparatively small values of the parameter $\mathrm{r} \approx 0.22 \mathrm{fm}$ and the numerical values of those parameters are slightly different in these two regions
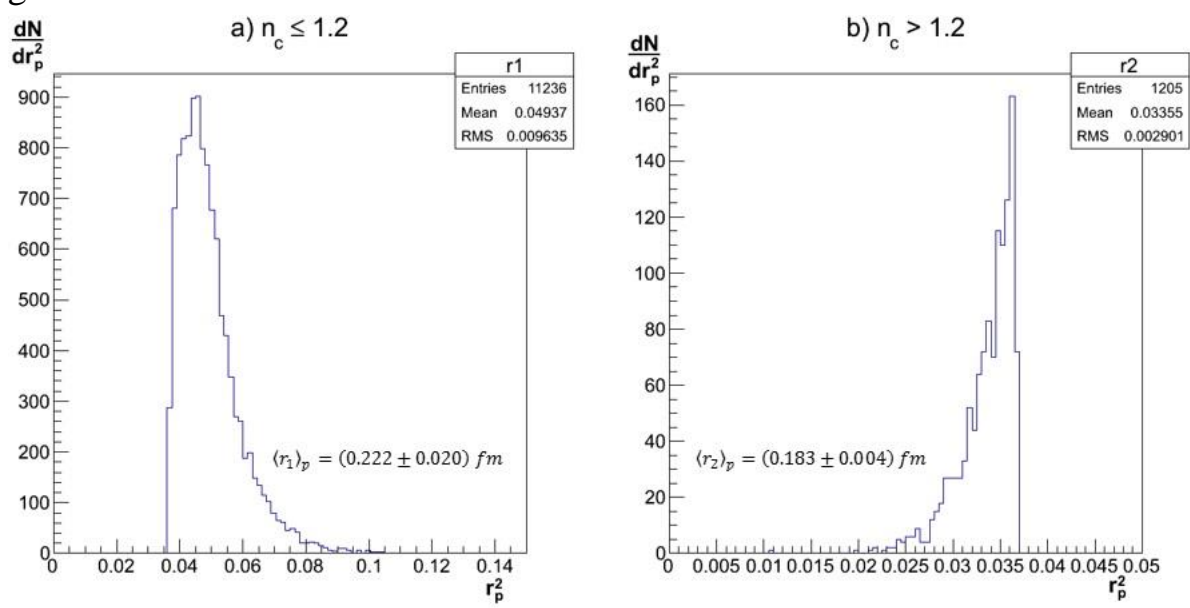

Fig. 9. (a, b) Distributions on the parameter $r^{2}$ for protons in different $n_{c}$ regions

Average value of the parameters $\left\langle r^{2}\right\rangle_{p},\langle r\rangle_{p}$ for all protons and protons in two different regions on the variable $n_{c}$ are given in Table 3 .

We would like to note that the average values of the parameter $\langle r\rangle$ are essentially different not only for all $\pi^{-}$mesons and protons, but also for $\pi^{-}$mesons and protons produced in different $n_{c}$ regions corresponding to different phase.

Table 3

\begin{tabular}{|c|c|c|c|}
\hline \multicolumn{4}{|c|}{$\pi^{-}+C \rightarrow p+X$} \\
\hline & All protons & Protons with $\mathrm{n}_{\mathrm{c}} \leq 1.2$ & Protons with $\mathrm{n}_{\mathrm{c}}>1.2$ \\
\hline$N_{p}$ & 12441 & 11236 & 1205 \\
\hline$\left\langle r^{2}\right\rangle_{p}\left(\mathrm{fm}^{2}\right)$ & $0.04778 \pm 0.00009$ & $0.04937 \pm 0.0046$ & $0.03355 \pm 0.00008$ \\
\hline$\langle r\rangle_{p}(\mathrm{fm})$ & $0.21858 \pm 0.00041$ & $0.2222 \pm 0.0020$ & $0.1832 \pm 0.0004$ \\
\hline
\end{tabular}

\subsection{Average values of the proton momentum $<P>_{p}$ on the variable $n_{c}$}

The distribution on the variable $n_{c}$ for the secondary protons from $\pi^{-} C$ interactions is presented on Fig.10. From this figure we see that the maximum of the distribution is at $n_{c} \approx 1$ and $\sim 60 \%$ of protons are produced in the region $n_{c} \leq 1$ and $\sim 40 \%$ of protons are produced in the $n_{c}>1$ region. So $40 \%$ of protons are produced in the cumulative particle production region $n_{c}>$ 1. 


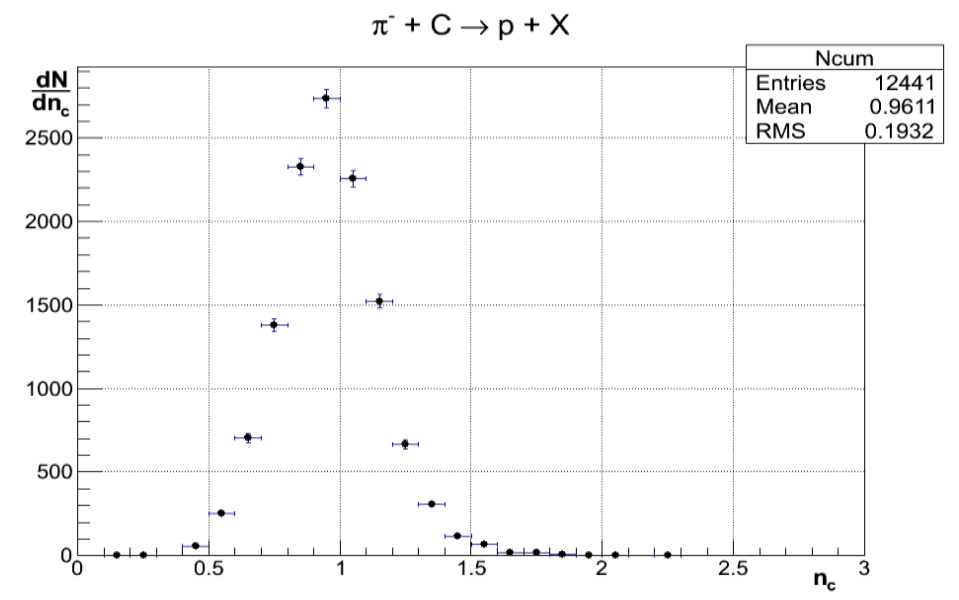

Fig.10. Cumulative number $\left(n_{c}\right)$ distribution of protons. This figure was taken from [1]

Fig 11 presents the dependence of the average momentum of protons as a function of variable $n_{c}$. With increasing $n_{c}$ average values of the momentum $\langle P\rangle_{P}$ are decreased and reach the minimum at $n_{c} \approx 1$ and then in the cumulative particle production region $\left(n_{c}>1\right)$ are essentially increased. In addition to this we note that average values of the transverse momentum square $\left\langle P_{t}^{2}\right\rangle$ (see [1]) and the effective temperature T (Fig.7) are remained practically constant in the region $r \geq \lambda_{c}^{p}=0.21 \mathrm{fm}$, but in the region $r<\lambda_{c}^{p}$ we see the reversed feature, in other words, $\langle P\rangle_{p},\left\langle P_{t}^{2}\right\rangle$ and $\mathrm{T}$ are essentially increased. So, the essentially different features of the above mentioned characteristics $\langle P\rangle_{p},\left\langle P_{t}^{2}\right\rangle$ and $\mathrm{T}$ in these two regions $\left(r \lesssim \lambda_{c}^{p}\right.$ and $\left.r>\lambda_{c}^{p}\right)$ as mentioned in the previous paper [1] indicate about the particle production different mechanism.

The numerical value of the parameter $\mathrm{r}$ at $\mathrm{n}_{\mathrm{c}} \approx 1$ was shown by arrow on this figure.

From the other hand side, it is well known that if a particle is localized in the region $r<$ $\chi_{c}^{P}=0.21 \mathrm{fm}$, then this particle is regarded as a "not point like object" and its interaction should be described by Quantum field Theory (QFT)[8]. So, the essentially different behaviors of the proton's characteristics indicate about the separation of protons produced as a result of soft and hard processes.

$$
\pi^{-}+\mathrm{C} \rightarrow \mathrm{p}+\mathrm{X}
$$

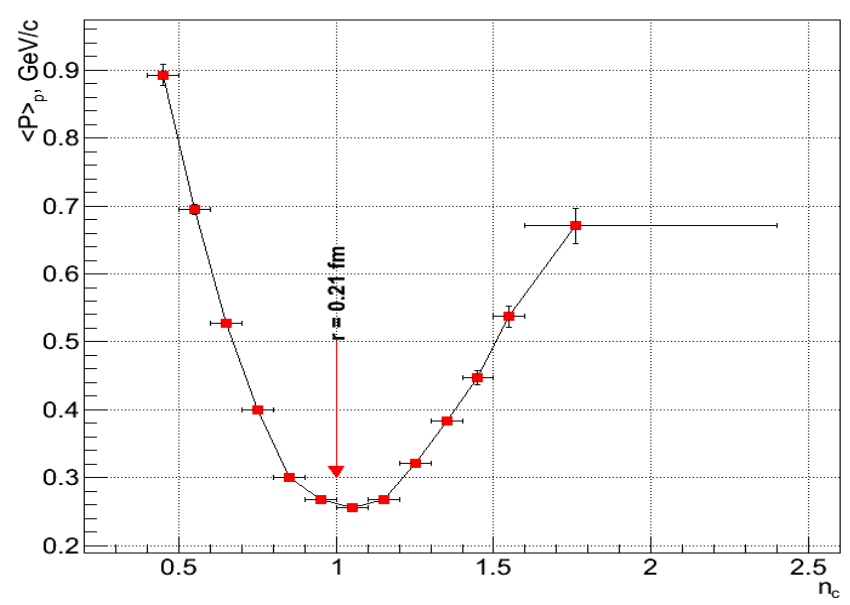

Fig.11. The average values of the momentum of the secondary protons as a function of the variable $n_{c}$. This figure was taken from paper[1] 


\section{Conclusion}

In this paper we are obtained the formula which determines the particle emission region size $r$.

$$
r=\frac{1}{m_{p} \sqrt{1+\frac{2 E_{a} \cdot m_{p}\left(n_{c}-1\right)}{2 E_{a \cdot} \cdot m_{p}-\left(m_{a}^{2}+m_{c}^{2}\right)}}} \approx \frac{1}{\sqrt{n_{c}} \cdot m_{p}}=\frac{\lambda_{c}^{P}}{\sqrt{n_{c}}}=\frac{0.21 \mathrm{fm}}{\sqrt{n_{c}}}
$$

The distribution on the parameter $r$ calculated by the formula (8) and their average values $\sqrt{\langle r\rangle^{2}}$ are presented for the all secondary $\pi^{-}$mesons and protons and also for $\pi^{-}$mesons and protons corresponding to the different phase transition regions from $\pi^{-} C$ interactions at 40 $\mathrm{GeV} / \mathrm{c}$.

\section{Acknowledgement}

We would like to express our thanks to Kh.Namsrai, G.Ganbold and Ts.Enkhbat for their valuable discussions and remarks.

\section{References}

[1] Ts. Baatar, et al. JINR, E1-2012-13

[2] Baldin A.M. Particles and Nuclei, 1977, 8p, p.429

[3] Dirk H.Rischke, arXiv:nucl-th/0305030v2

[4] D.J. Gross and F.Wilczek, Phys. Rev.Lett. 30(1973) 1343

[5] Ts.Baatar et al, JINR, pl-6937, Dubna, 1973

[6] Ts.Baatar et al, Journal of Nuclear Physics, 52, 3(9),1990

[7] Ts.Baatar et al, In proceeding of XXI International Baldin Seminar on High Energy Physics, JINR, Dubna, Russia, September 10-15, 2012

[8] Physical Encyclopedia, v.2, p.433, Moscow, 1990

[9] P.J. Mohr, B.N.Taylor and D.B.Newell(2011) "The 2010 CODATA Recommended Values of the Fundamental Physical constants" National Institute of Standards and Technology, Gaitherburg, MD, US 